\title{
Ueber generative und embryonale Mitosen, sowie über pathologische Kerntheilungsbilder.
}

\author{
Von \\ Dr. Valentin Hacker, \\ Privatdozent und Assistent am zoologischen Institut der Universität \\ Freiburg i. Br.
}

Hierzu Tafel XXXII und 2 Figuren im Text.

Die neueste Arbeit vom Rath's') hat werthvolle Beiträge für die Kenntniss derjenigen besonderen Kerntheilungsformen geliefert, welche vorzugsweise in Cyklus der generativen Zelien auftreten und sich als "plurivalente Kerntheilungen" zusammenfassen lassen. Ich nehme die Gelegenheit wahr, unter Heranziehung einiger nicht veröffentlichter Beobachtungen noch einmal auf den genetischen Zusammenhang der plurivalenten (generativen) mit den gewöhnlichen (somatischen) Mitosen zurückzukommen.

Nachdem vom Rath in einer vorläufigen Mittheilung (1891) die Entstehung der bekannten "Vierergruppen" der Reifungsperiode aus Ringen beschrieben hatte, sprach er sich in seiner ausführlichen Arbeit $^{2}$ ) dahin aus, dass jedes der sechs Doppelfadensegmente, aus welchen die eigenthümlichen Chromatinringe und später die „Vierergruppen" hervorgehen, „zwei Segmenten homolog gesetzt werden kann" (S. 110). Darnach nahm also vom Rath an, dass die für die Spezies typische Theilungszahl "12" auch hier, wenigstens implicite, vorhanden ist.

Die Kerntheilungsvorgänge, welche die Urgenitalzellenbildung von Cyclops einleiten und bei welchen eine Doppelwerthig-

1) 0. vom Rath, Beiträge zur Kenntniss der Spermatogenese von Salamandra maculosa. Zeitschr. f. wiss. Zool. 57. Bd. 1893. (1.)

2) O. vom Rath, Zur Kenntniss der Spermatogenese von Gryllotalpa vulgaris Latr. Arch. f. mikr. Anat. 40. Bd. 1892. (2.)

Archiv f. mikrosk. Anat. Bd. 43 
keit der Chromosomen wirklich bewiesen werden konnte, sind für mich einige Zeit nachher (1892) ${ }^{1}$ ) der Ausgangspunkt fitr einen Versuch gewesen, sämmtliche im Cyklus der generativen Zellen auftretenden Abarten der Mitose unter einem einheitlichen Gesichtspunkt zusammenzufassen. Darnach würde das besondere Wesen dieser Theilungsformen darin begründet sein, dass sie bezüglich der Zerlegung des Doppelfadens im Segmente um eine Stufe gegenüber den somatischen Mitosen zurückbleiben. Nebmen wir beispielsweise eine Spezies mit der Theilung\&zahl "12" an: dann wird sich in den betreffenden Mitosen des generativen ZellenCyklus der Doppelfaden nur in sechs Segmente zerlegen, aber die Einzelfäden dieser Doppelfadensegmente sind doppelwerthig, indem sie aus zwei hintereinander gereihten einfachen Elementen bestehen. Es tritt also auch hier, obschon in verdeckter Weise, die normale Theilungszahl auf und dies kommt in einzelnen Fällen in der nachträglichen Halbirung der an die Pole rü ckenden Schleifen zum handgreiflichen Ausdruck. In Uebereinstimmung mit vom Rath glaubte ich auch die "Vierergruppen" dahin deuten zu können, dass je zwei im ursprünglichen Spirem hintereinander liegende Segmente (Kugelchromosomen) zunächst in engerem $\mathrm{Zusammenhang}$ bleiben und sich mit dem entsprechenden (durch Längsspaltung entstandenen) Schwesterpaar zu einem ringförmigen, viertheiligen Gebilde vereinigen. Des weiteren kam ich zu dem Schluss, dass auch bei der im Salamanderhoden auftretenden "heterotypischen" Theilungsform die Sehleifen aus je zwei hintereinander liegenden Chromosomen bestehen, ferner dass die im Dyaster dieser Theilung bemerkliche Längsspaltung eine vorzeitige, auf die nächstfolgende Theilung sich beziehende Längsspaltung darstellt, und endlich, dass die von Flemming im Salamanderhoden gefundenen und als abnorme Vorkommnisse gesehilderten Vierergruppen der ersten Theilung der Reifungsphase angehören. Ich fasste damals alle Theilungen mit doppelwerthigen Elementen unter der Flemming'schen Bezeichnung der "heterotypischen" Theilungsform zusammen, sah mich aber aus praktischen Gründen bald darauf veranlasst, diesem Ausdruck seine ursprüngliche Be-

1) V. Häcker, Die heterotypische Kerntheilung im Cyklus der generativen Zellen. Ber. Nat. Ges. Freiburg. 6. Bd. 1892. (3.) 
deutung zu lassen, und jene Theilungen als Theilungen mit plurivalenten (streng genommen bivalenten) ${ }^{1}$ ) Chromosomen oder kurz als "plurivalente (bivalente) Theilungen" zusammenzufassen.

Bald darauf ${ }^{2}$ ) gelang es mir, für die Eizellen von Cyclops strenuus den Nachweis zu führen, dass die Bildung der Vierergruppen (und damit die erste Richtungstheilung) dem plurivalenten Schema folgt. Im besondern wurde ihre Entstehung ans ${ }_{n}$ Doppelwinkeln" dargelegt und betont, dass ihrer Bildung nur eine e inma lige Längsspaltung des Chromatinfadens vorangeht.

In seiner neuen Arbeit über die Spermatogenese von Salamandra (1) hat nunmehr vom $R$ at h dieselbe Entstehungsweise der Vierergruppen in erster Linie für Salamandra, dann aber noch für weitere Formen nachgewiesen. Seine Figur 7 (1, Taf. VII) stimmt in allen wichtigen Punkten mit meiner Figur 16 (4, Taf. XXVII) und meinem Schema A (4, S. 482) ïberein. Vom Rath gelangte ausserdem seinerseits zu der Auffassung, dass sowohl die heterotypen als die homöotypen Theilungen im Salamanderhoden doppelwerthige Elemente haben müssen (l. c. S. 121) und stellte endlich noch fest, dass die Längsspaltung: der Schleifen im Dyaster der heterotypen Theilung wirklich eine vorzeitige Lüngsspaltung für die nächstfolgende Theilung darstellt (l. c. S. 109) und demnach mit der im Dyaster der letzten Theilung der Ureizellen von Cyclops auftretenden vorzeitigen Längsspaltung verglichen werden kann (I. c. S. 119).

Während so die empirischen Befunde, ebenso wie die darauf gegründeten Anschanungen vom Rath's in allen wesentlichen Punkten mit den meinigen im Einklang sind, scheint ihm meine Bezeichnung "plurivalente Mitosen" für Theilungen mit doppelwerthigen Elementen nicht besonders glticklich zu sein und er möchte lieber vorläufig den Ausdruck „halbzählige Mitosen" verwenden. Was vom Rath unter ",halbzähligen" Mitosen versteht, darauf weisen folgende beide Citate hin. "Die homöotypen und heterotypen Theilungen der Ursamenzellen des Salamanders folgen beide einer Mitose mit doppelwerthigen Schleifen

1) Der Ausdruck „bivalent“, welcher am besten geeignet gewesen wäre, hat durch 0 . Hertwig bereits eine Verwendung in entgegengesetztem Sinne gefunden (Ascaris megalocephala bivalens).

2) V. Häcker, Das Keimbläschen, seine Elemente und Lageveränderungen. I. Arch. f. mikr. Anat. 41. Bd. 1893. (4.) 
(= halbzählige Mitose) — (1. c. S. 109). „In einer eben erschienenen neuen Arbeit lässt nun Hä cker die Verallgemeinerung des Begriffes der heterotypen Theilung wieder fallen und vereinigt alle Varianten mit doppelwerthigen Segmenten unter der leider auch nicht besonders glücklichen Bezeichnung von "plurivalenten" Kerntheilungen, da der viel sachgemässere Ausdruck bivalens schon in einem anderen Sinne Verwendung gefunden hat. Vielleicht wäre die Bezeichnung halbzählige Mitosen einstweilen zu verwenden" (l. c. S. 124). Daraus würde wohl zunächst zu entnehmen sein, dass vom Rath's Ausdruck ,halbzählige Mitosen" sich dem Sinne nach mit dem Ausdruck "plurivalente Mitosen" decken soll.

Ich fürchte aber, dass durch eine doppelte Nomenclatur in den an und für sich schon schwierigen Gegenstand Verwirrung hereingetragen wird, und möchte daher die Gründe darlegen, welche mir gegen die Anwendbarkeit des Ausdrucks ,halbzählig“ a $\mathrm{Stelle}$ der von mir vorgeschlagenen Bezeichnung zu sprechen scheinen. Zunächst glaube ich, dass der Ausdruck „halbzählig" in keiner Weise den Kern der Sache trifft. Denn unter ",halbzähligen" Mitosen wird man wohl zunächst nur Theilungen mit der halben Zahl von Elementen verstehen. Vom Rath und ich legen aber doch gerade darauf Werth, dass die betreffenden Mitosen nur seheinbar die halbe Zahl von Elementen besitzen, thatsächlich aber in Folge der Doppelwerthigkeit der Chromosomen ganzzählig sind. Der Ausdruck „halbzählig" wäre also offenbar ein lucus a non lucendo, falls er im Sinne von „plurivalent" gebraucht würde.

Es spricht nun aber ausserdem gegen die Verwendung des Ausdrucks ,halbzählig" ein weiterer, und wie mir scheint, besonders schwerwiegender Umstand. Es giebt nämlich eine Reihe von Mitosen, welche thatsä chlieh nur die halbe Zahl von Elementen besitzen, die aber doch nicht unter den Begriff der „Halbzähligkeit" im Sinne der obigen beiden Citate fallen. Hierher gehören 7. B. die Theilungen selbstständiger Spermakerne in eikernlosen Fragmenten der Seeigeleier (Boveri $5^{1}$ ), S. 345), ferner die Theilungen der Merocytenkerne im Selachierei, bei

1) Th. Boveri, Zellen-Studien III. Jen. Zeitschr. 24. Bd, 1890. (5.) 
welchen nach Rückert $\left(6^{1}\right)$, S. 329) halb so viel Elemente als in den Furchungskernen auftreten und welche dieser Autor daher für Abkömmlinge von Spermaköpfen hält. Halbzählig im eigentlichen Sinne des Wortes ist endlich aber vor Allem auch die zweite Theilung der Reifungsphase sowohl bei der Spermato. genese, als bei der Orogenese. Denn bei derselben sind nach den ïbereinstimmenden Befunden der meisten Autoren von vornherein nur halb so viel Chromosomen vorhanden, als der normalen Theilungszahl der betreffenden Spezies entsprechen würde. Hier haben wir also thatsächlich Theilungen mit der halben Zahl von Elementen vor uns, die aber trotzdem im Sinn der vom $\mathrm{R}$ ath 'schen Citate nicht als "halbzählig" bezeichnet werden dürfen.

Angesichts des Umstandes, dass der Ausdruck ,halbzählig“ dem Wortlaut nach offenbar nicht eindeutig ist und demnach wohl kaum als eine Verbesserung des früheren Ausdrucks ,plurivalent" zu bezeichnen ist, möchte ich den Vorschlag machen, den Ausdruck ,halbzählig“ beizubebalten, ihm aber entgegen der v $0 \mathrm{~m} R$ at $\mathrm{h}$ 'schen Definition einen allgemeineren Sinn zu unterlegen.

Als „halbzählige" Mitosen möchte ich ohne Rü cksicht auf irgend welche theoretischen Vorstel$\mathrm{lu} \mathrm{ng}$ en alle diejenigen Theilungen bezeichnen, in denen eine Anzahl von Theilungseinheiten (Chromosomen) auftritt, welche der Hälfte der „Normalzahl" entspricht. Die „Normalzahl“ würde dabei, wenn möglich, den Soma-Mitosen des ausgewachsenen Thieres zu entnehmen sein. „Halbzählig“" würden demnach ebensogut die zweite Reifungstheilung und die Theilungen isolirter Spermaköpfe sein, wie diejenigen Mitosen, für welche eine Doppelwerthigkeit der Elemente bewiesen oder theoretisch wahrscheinlich gemacht werden kann.

1) J. Rückert, Ueber physiologische Polyspermie bei meroblastischen Wirbelthiereiern. Anat. Anz. 7. Jahrg. 1892. (6.) H. E. Ziegler hält dagegen die Ableitung der Merocytenkerne von Nebenspermakernen nicht für bewiesen und stellt die betreffenden Mitosen hinsichtlich der Zahl der Chromosomen den weiter unten zu besprechenden Befunden vom Rath's bei Salamandra-Embryonen und -Larven an die Seite. Vergl. H. E. Ziegler, Ueber das Verhalten der Kerne im Dotter der meroblastischen Wirbelthiere. Ber. Nat. Ges. Freiburg. 8. Bd. 1894 (Festschrift für A. Weismann). (6a.) 


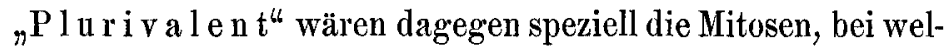
chen die geringere Anzahl der Theilungselemente auf eine Doppeloder Mehrwerthigkeit ${ }^{1}$ ) der Elemente zurückzuführen ist. D a s plurivalente Schemaversucht im Speziellen eine Erklärung zu geben für das Auftreten von Mitosen mit halber Chromosomenzahl innerhalb des Cyklus dergenerativen Zellen und insbesondere auf die Entstehung der Vierergruppen und die Bedeutung der heterotypen Theilung ein Licht zu werfen ${ }^{2}$ ).

Ich hoffe, dass diese Fassung der Begriffe eine sachdienliche und annehmbare ist.

Aus dem Bisherigen geht hervor, dass $\mathrm{v} o \mathrm{~m} \mathrm{R}$ ath und ich bezuiglich der Entstehung und Bedeutung der Vierergruppen zu den nämlichen Ergebnissen gelangt sind. Da die Bildungsweise dieser Theilungselemente von theoretischer Wichtigkeit ist und da dieselben nach den tubereinstimmenden Angaben der meisten neueren Autoren eine allgemeine Verbreitung haben, so möchte ich auf Grund des reichen und vielseitigen, meinem Arbeitsgenossen vom Rath und $\mathrm{mir}$ vorliegenden Materiales den Versuch machen, auch die Angaben anderer Forscher von dem von uns gewonnenen Gesichtspunkt aus zu prüfen. Es soll dabei nur auf solche Arbeiten eingegangen werden, in welchen eben die Frage nach der Entstehung der Vierergruppen erörtert worden ist.

Für das Problem der Vierergruppen besteht offenbar nur das Dilemma: entstehen dieselben durch einmalige oder zweimalige Längsspaltung? Vom $R$ ath und ich sind zu den über-

1) Eine Zusammensetzung der Schleifen aus mehr als zwei Theilungseinheiten niedrigerer Ordnung scheint, wie unten gezeigt werden soll, bei Ascaris vorzukommen. Der allgemeinere Ausdruck "plurivalent" dürfte daher schon an und für sich den Vorzug vor der näherliegenden Bezeichnung "bivalent" verdienen.

2) Beispielsweise würden die von vom Rath gefundenen Mitosen in embryonalen und larvalen Geweben von Salamandra, welche anstatt der Normalzahl 24 nur 12 Elemente besitzen (l. c., S. 106, Anm.), zunächst als "halbzählig" zu bezeichnen sein. Indem aber vom Rath die Aehnlichkeit derselben mit der homöotypen Form hervorhebt und an die Möglichkeit einer Doppelwerthigkeit der Schleifen denkt, weist er diesen Mitosen irn Speziellen auch einen „plurivalenten" Charakter zu. 
einstimmenden Resultate gelangt, dass nur eine einmalige Längsspaltung vorliegt, dass aber je zwei hintereinanderliegende Segmente zunächst miteinander verbunden bleiben. Es entstehen so Doppelwinkel, aus welchen unter Durchbruch der Winkelecken (Metalyse) und Parallellagerung der sich verkürzenden Stäbchen die Vierergruppen hervorgehen. Von den vier Elementen sind also je zwei einander identisch ${ }^{1}$ ). Wenn nun, was bis jetzt in einem einzigen Fall beobachtet wurde (4, S. 485), bei der ersten Theilung die an die Pole riickenden $Z$ weiergruppen je einem Einzelwinkel entsprechen, also je zwei nicht-identische Nachbar-Elemente darstellen, so haben wir anch hier das plurivalente Schema vor uns, insofern zwei in Spirem hintereinander gelegene Elemente zunächst miteinander im engeren Verbande bleiben. In der zweiten Theilung würde dann die endgültige Trennung der nicht-identischen Paare, gewissermaassen durch Quertheilung erfolgen.

Im Gegensatz dazu haben Boveri und Brauer eine zweimalige Längsspaltung behauptet. Was zunächst die Bilder, welche B overi (5) von der ersten Richtungstheilung verschiedener Formen gibt, anbelangt, so wird aus dem Folgenden hervorgehen, dass sie zum Theil wenigstens auf eine Entstehung der Vierergruppen durch e in ma $\mathrm{l}$ ige Längsspaltung, beziehungsweise auf ihre Bildung nach dem plurivalenten Schema, hinweisen. Ich gebe hier zwei Bilder Boveri's wieder, von denen das erste (Boveri's Figur 3) die erste Richtungsspindel des Pterotrachea-Eies, das zweite (Boveri's Figur 13) die Vierergruppen des Carinaria-Eies darstellt ${ }^{2}$.

In Figur 3 sehen wir die an die Pole rückenden Elemente theils in Form paarweise angeordneter Stäbchen, theils als winklig gebogene Schleifen auftreten: Boveri zählt auf jeder Seite 16 Chromosomen, indem er die Einzelstäbchen und die Schleifen als gleichwerthige Gebilde ansieht. Nimmt man aber, unter Be-

1) Unter identischen Elementen versteht Weismann soiche Elemente, die durch Längsspaltung eines Mutterelementes entstanden sind (Schwesterelemente), ni cht identisch sind dagegen zwei im Faden hintereinander gelegene Elemente (Nachbarelemente).

2) Die beiden Bilder sind durch einen hiesigen Lithographen, Herrn Schilling, nach den Boveri'schen Figuren copirt und für die zinkographische Darstellung vergrössert worden. 
rücksichtigung der Befunde von mir und vom $R$ ath, die schleifenförmigen Gebilde als doppelwerthig, die Einzelstäbchen als einwerthig an, so hat man das Bild einer plurivalenten Mitose vor
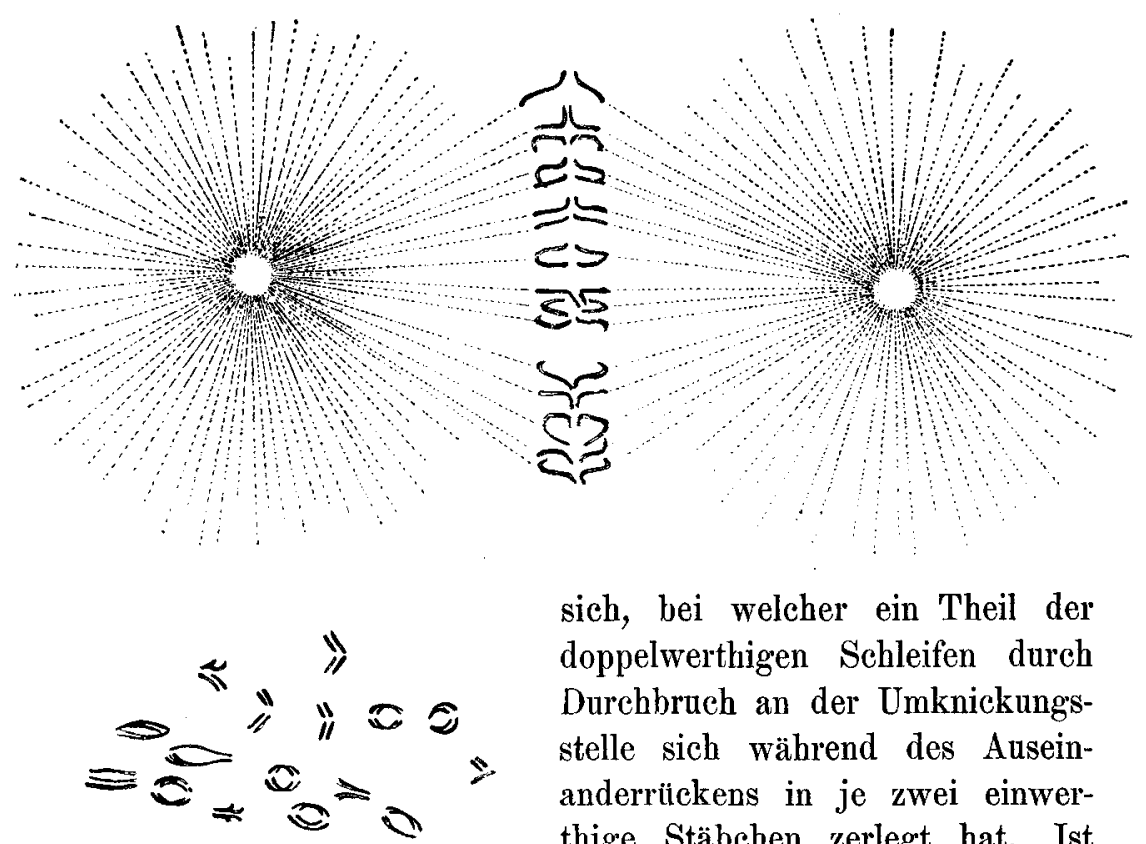

sich, bei welcher ein Theil der doppelwerthigen Schleifen durch Durchbruch an der Umknickungsstelle sich wäbrend des Auseinanderriickens in je zwei einwerthige Stäbchen zerlegt hat. Ist dies richtig, so folgt mit Nothwendigkeit, dass die Vierergruppen, welche hier aus stäbchen- und nicht aus kugelförmigen Einzelgebilden zusammengesetzt sind, einer e in $\mathrm{m}$ a l i g e $\mathrm{n}$ Längsspaltung und nachfolgenden Knickung und Quertheilung der Segmente ihre Entstehung verdanken. Allerdings muss dann im Hinblick auf die Normalzahl angenommen werden, dass Boveri speciell bei der Figur 3 das eine oder andere Chromosomenpar übersehen hat, eine Möglichkeit, welche angesichts der grossen Schwierigkeit aller Chromosomenzählungen wohl eingeräumt werden muss.

Die Figur 13 ist gleichfalls von B overi im Sinn einer zweimaligen Längsspaltung gedeutet worden. Ein Theil der Chromosomengruppen besitzt aber offenbar eine grosse Aehnlichkeit mit den "Doppelwinkeln", welche von mir und vom Rath als Anfangsstadien der Vierergruppen festgestellt worden sind. Man könnte also auch für Carinaria annehmen, dass die Vierergruppen durch einmalige Längsspaltung des Fadens, durch Um- 
knickung der anfänglich entstandenen Segmente (Doppelwinkelbildung) und nachtrïglichen Durchbruch der Winkelecken (Metalyse) ihre Entstehung nehmen ${ }^{1}$ ).

Man könnte ferner geneigt sein, auch die in der Aequatorialplatte der ersten Richtungsspindel von Sagitta (Boveri, Fig. 17) auftretenden Doppelwinkel im Sinne einer plurivalenten Theilung aufuufassen. So viel dürfte jedenfalls aus dem Obigen hervorgehen, dass die Boveri'schen Bilder zum Theil nicht nur eine Interpretation im Sinne meiner Annahme zulassen, sondern dass sogar gewisse Einzelheiten derselben nur durch die letztere verständlich werden. Der Beweis einer $z$ w eimaligen Längsspaltung scheint mir aber andererseits von Boveri nicht geliefert worden zu sein.

Was die Pyrrhocoris betreffenden älteren Bilder Henking's anbelangt, so haben dieselben bereits von Seiten Weis mann's (Amphimixis, Jena 1891) eine Deutung erfahren, welche mit der hier vorgetragenen Auffassungsweise übereinstimmt. In der neueren Arbeit Henkin g's $^{2}$ ) sind nur in wenigen Figuren (beispielsweise Fig. 101 und 104) Vierergruppen andeutungsweise wieder gegeben worden. Dass dieselben aber auch bei Insekten verbreitet sind, ist durch vom $R$ ath in unzweifelhafter Weise dargethan worden.

Die von Brauer ${ }^{3}$ ) für das Branchipus-Ei gegebene Darstellung ist neuerdings durch vo m $R$ at $h(1, S .135)$ besprochen und berichtigt worden. Während $\mathrm{Br}$ r u e r eine Entstehung der Vierergruppen durch zweimalige Längsspaltung annimmt, kommt vo m Rath auf Grund eigener Beobachtungen zu Ergebnissen, welche unsern anderweitigen Befunden vollkommen entsprechen.

Hinsichtlich des oben (S. 764) aufgestellten Dilemmas steht also auf der einen Seite eine Reihe von Befunden, welche über-

1) Boveri lässt die Figur 13 dem Stadium entsprechen, in welchem die "primären" (auf die erste Theilung bezüglichen) Schwesterelemente, deren jedes aus 2 scharf geschiedenen parallelen (sekundären) Fädchen besteht, gerade begonnen haben, sich von einander zu entfernen.

2) H. Henking, Untersuchungen über die ersten Entwicklungsvorgänge in den Eiern der Insekten. III. Spezielles und Allgemeines. Zeitschr. f. wiss. Zool. 54. Bd. 1892. (7.)

3) A. Brauer, Ueber das Ei yon Branchipus Grubii. Abhandl. d. k. preuss. Ak. d. Wiss. Berlin 1892. (8.) 
haupt nur in der durch mich and vo $\mathrm{m} R$ at $\mathrm{h}$ vertretenen Weise gedeutet werden können, und eine Anzahl anderer, welche wenigstens eine Interpretation in unserem Sinne zulassen und nahelegen (Boveri's Molluskenbefunde). Es ist auch gleich hier zu erwähnen, dass bei der Bildung der Vierergruppen zwei Haupttypen $\mathrm{zu}$ unterscheiden sind. Im einen Fall gehen aus den Doppelwinkeln unter tropfenförmiger Anschwellung der Winkelenden „Kugelchromosomen" hervor, im andern bleiben die vier Schenkel der Winkel als "Stäbchenchromosomen" erhalten und legen sich nach Durchbruch an der Umknickungsstelle - vermuthlich in Folge gegenseitiger Anziehung - parallel zu einander zu einem Bündel zusammen.

Dieser Uebereinstimmung gegenüber stehen nun in schroffem Gegensatz die Brauer'schen Befunde bei der Spermatogenese von Ascaris ${ }^{1}$ ). Hier wird mit aller Bestimmtheit eine zweimalige Längsspaltung des Chromatinfadens vor der Bildung der Vierergruppen behauptet und wir stehen also damit vor der Wahl, entweder in diesem Punkte eine tiefgreifende Verschiedenheit innerhalb des Metazoenreiches anzunehmen, oder die Deutung, welche Bra uer seinen Befunden gibt, für unrichtig zu halten. Eine im hiesigen zoologischen Institut in Ausführung begriffene Arbeit wird auf das Verhältniss der bei Ascaris auftretenden Bilder mit den übrigen Beobachtungen abermals eingehen. Aus den mir selbst vorliegenden Ascaris-Präparaten geht jedenfalls soviel hervor, dass bezüglich der einschlägigen Stadien die Bilder bei Ascaris an Klarheit hinter Gryllotalpa, Salamandra und den Copepoden zurïckstehen, und dass wohl auch durch B raner's Arbeit die Frage nach den Reifungstheilungen von Ascaris nicht eine definitive Erledigung gefunden hat.

Schon aus allgemeineren Gründen von mehr theoretischer Natur würde es übrigens in hohem Maasse wahrscheinlich sein, dass auch die Ascaris-Bilder mit den Befunden bei andern Formen im Einklang stehen.

Bekanntlich hängt die Frage nach der Entstehung der Vierergruppen mit der "Reductionsfrage" auf's Engste zusammen. Dies ist erst vor Kurzem durch vom $R$ ath ausführlich erörtert

1) A. Brauer, Zur Kenntniss der Spermatogenese von Ascaris megalocephala. Arch. f. mikr. An. 42. Bd. 1893. (9.) 
worden und es bleibt mir hiel nur noch übrig, auf einen Punkt hinzuweisen. Ueberall wo die Zahlenverhältnisse der Chromosomen klar zu Tage liegen, vor Allem bei Ascaris, Cyclops und Salamandra, tritt die gemeinsame Erscheinung hervor, dass unmittelbar vor der ersten Richtungstheilung doppelt so viele $f$ ertige Theilungseinheiten auftreten, als die "Normalzahl“ der Spezies beträgt. Es finden sich also hier, was die Zahl der fertigen, zur Vertheilung bereiten Elemente anbelangt, genau die nämlichen Verhältnisse, wie bei jeder gewöhnlichen Mitose. Durch zweimalige Vertheilung findet die Herabsetzung dieser Anzahl auf die Hälfte der Normalzahl statt, so wie es von Weismann theoretisch postulirt wurde.

Boveri muss nun bekanntlich, um seine zweimalige Längsspaltung aufrecht erhalten zu können, annehmen, dass bereits im $\mathrm{R} \mathfrak{u} \mathrm{h}$ estadi um diese Reduction der Chromosomenzahl auf eine nicht weiter erkennbare Weise stattfindet. Dies steht aber mit der bei zoologischen Objecten anscheinend allgemein gültigen Regel in Widerspruch, welcher zu Folge die Anzahl der ans dem Ruhestadium hervorgehenden Mutterelemente der Anzahl der Tochterelemente entspricht, welche nach Ablauf der vorangegangenen Theilung in das Ruhestadium eingegangen waren. Diese Regel ist bis jetzt a uf zoologischem Gebiet durch keine Thatsache umgestossen worden, denn in denjenigen Fällen, in welchen im Cyklus der generativen Zellen eine halbe Anzahl von Chromosomen auftritt, konnte eine Bivalenz der Elemente theils bewiesen (Urgenitalzellen und Ureizellen von Cyclops), theils wahrscheinlich gemacht werden. Auf die einzige Beobachtung, welche gegen die erwähnte Regel zu sprechen scheint, nämlich auf die Boveri'schen Befunde bei den Furchungskernen von Ascaris, werde ich im Schlussabschnitt zu sprechen kommen: ich werde dort an der Hand eigener Beobachtungen zu zeigen versuchen, dass dieselben sich durchaus innerhalb des Rahmens der bisher bekannten Thatsachen bewegen. Alles in Allem scheint mir heute die obige Regel fester als je zu stehen, so dass sie vielleicht sogar zu einem Gesetz der konstanten Idantenzahl ${ }^{1}$ ) er-

1) Ich wähle hier den Weismann'schen Ausdruck "Idant", um den Ausdruck "Chromosom" für die bei den verschiedenen Kern- 
hoben werden darf, und die von Rabl und Boveri begründete, von Boveri selbst aber wieder ins Schwanken gebrachte „Individualitätshypothese" würde damit wieder eine sicherere Basis erhalten. Es ist indess hier nicht meine Absicht, auf die theoretische Bedeutung dieses Gesetzes einzugehen, ich habe vielmehr diese Verhältnisse aus einem andern Grunde herangezogen. Ich wollte damit zeigen, dass die Brauer'sehen Befunde bei Ascaris nicht nur mit den ubrigen Thatsachen der Ei- und Samenreife, sondern mit ganz allgemeinen kerntheilungsgeschichtlichen Prinzipien im Widerspruch stehen, falls nicht eine andere Deutung derselben zulässig ist, als die von Brauer selbst gegebene.

In einer Reihe von kürzeren Mittheilungen ${ }^{1}$ ) hat uns Boveri mit einem Vorgang bekannt gemacht, der sich in den Furchungskernen von Ascaris meg. abspielt und auf einen Gegensatz der chromatischen Substanz der Sexualzellen und jener der somatischen Zellen hinweist. Im Zweizellenstadium von Asc. meg. univalens gehen in jedem der beiden Kerne aus dem Ruhestadium je zwei bandförmige, an den Enden verdickte Chromosomen hervor. Im Kerne der einen Zelle („erste Stammzelle“) ${ }^{2}$ )

theilungstormen thatsächlich wahrzunehmenden, morphologischen Theilungseinheiten $\mathrm{zu}$ reserviren.

Boveri selbst stellte früher (5, S. 372) das Gesetz auf: „Für jede Spezies ist die Zahl der Chromosomen konstant, d. h. in den karyokinetischen Figuren homologer Zellen finden sich stets die gleichen Zahlen." Dieses Gesetz lässt sich in dieser Fassung nicht mehr aufrecht erhalten. Dies zeigen vor Allem die in den ersten Furchungsstadien von Ascaris vorkommenden Variationen, welche Boveri beschrieben hat und auf welche weiter unten zurückgekommen werden soll.

1) Th. Boveri, Ueber Differenzirung der Zellkerne während der Furchung des Eies von Ascaris megalocephala. Anatom. Anzeig. 2. Jahrg. 1887. (10.) - Derselbe, Zellen-Studien III. (5.) S. 392 . Derselbe, Ref. „Befruchtung“ in Merkel und Bonnet, Ergebnisse der Anatomie und Entwicklungsgeschichte. I. Bd. 1891. (11.) S. 434. Derselbe, Ueber die Entstehung des Gegensatzes zwischen den Geschlechtszellen und den somatischen Zellen bei Ascaris meg. Sitz.-Ber. d. Ges. f. Morph. u. Phys. in München. 8. Bd. 1892. (12)

2) Der Uebersichtlichkeit zu liebe nummerire ich für den vorliegenden Zweck die Stammzellen sowohl als die Ursomazellen vom Zweizellenstadium an. Im Sinne Häckel's und Boveri's würde dagegen die befruchtete Eizelle als erste Stammzelle zu bezeichnen sein. 
spalten sich dieselben ihrer ganzen Länge nach in der für die Chromosomen des Einzellenstadiums beschriebenen Weise. Aus der folgenden Theilung gehen zwei grosse chromatinreiche Kerne hervor. Im Kern der zweiten Zelle des Zweizellenstadiums (,Ursomazelle I") machen sich zwei Eigenthümlichkeiten der chromatischen Substanz bemerklich, welche meiner Ansicht nach scharf auseinander zu halten sind:

Erstens werden von jedem der beiden Chromosomen die verdickten Enden abgestossen, dieselben bleiben zunächst im Aequator der Spindel liegen und gelangen schliesslich in das Innere der Tochterzellen, wo sie resorbirt werden.

Zweitens theilt sich der übriggebliebene mittlere Theil des Bandes in äusserst kleine Chromatinkörnchen, welche hier die sich spaltenden Theilungseinheiten darstellen.

Aus der Theiltmg des Kerns der Ursomazelle I gehen zwei kleine, chromatinarme Kerne herver. Im Vierzellenstadium sind also zwei Zellen mit grossen und zwei mit kleinen Kernen vorhanden. Beim Uebergang in das Achtzellenstadium treten in den beiden kleinen Kernen wieder die kleinen körnchenförmigen Theilungselemente auf, die beiden aus dem Kern der "Stammzelle" hervorgegangenen grossen Zellen verhalten sich nun ihrerseits wieder, wie die beiden ersten Furchungskerne. Nur in dem einen derselben, in dem Kern der "zweiten Stammzelle", treten die Chromatinstreifen als solche in die Theilung ein, im andern stellen sich wieder die Erscheinungen ein, welche im Kerne der Ursomazelle I beobachtet wurden. Dieser "Reductionsvorgang." (B overi) wiederholt sich noch dreimal und schliesslich bleibt nur noch eine Zelle mit ursprünglichem Kern, die Ur ges chlech tszelle, als direkter Abkömmling der Stammzellenreihe zurïck.

Betrachten wir nun zunächst den zweitgenannten Punkt, in welchem sich bei dem Boveri'schen Objecte die Somazellen von den Stamm- und Crgeschlechtszellen unterscheiden. Es tritt hier in unzweifelhafter Weise die Thatsache hervor, dass die Theilungselemente der Somakerne in grösserer Zahl und in Gestalt von kurzen, stäbchenförmigen Chromosomen auftreten, während bei den Stammzellen nur eine geringe Anzahl langer, band- oder schleifenförmiger Gebilde die Einheiten der Theilung: darstellt. Es ergibt sich so obne Weiteres eine Aehnlichkeit mit den Vorgängen, welche ich früher für die Keimblätterbildung: 
des Cyclops-Eies beschrieben habe: bei letzterem finden sich beim ersten Auftreten der Urgenitalzellen auffallend lange chromatische Gebilde, welche sich nicht nur durch ihre Grösse, sondern auch durch ihre geringere Anzahl von den Theilelementen der Furchungs- und Blastodermkerne unterscheiden. Die Figur 6 veranschaulicht den Gegensat\% im Kerntheilungshabitus, welcher bei Cyclops einerseits zwischen der Urgenital- (A) und Urmesodermzelle (B), den Abkömmlingen der "Stammzelle", andrerseits zwischen den Entoderm- (e) nnd Blastodermzellen besteht; dort lange, mannigfaltig gewundene und gedrehte Schleifen, hier kurze winkelförmige Gebilde. Die Figur 6 bildet ein vollkommenes Seitenstück zu den Figuren 2 und 4, welche B overi in seiner letzterschienenen Mittheilung (12) gegeben hat.

Es war bei Cyclops möglich, auf Grund gewisser, namentlich im Dyasterstadium auftretenden Erscheinungen den Schluss zu ziehen, dass jedes der Chromosomen der Urgenitalzelle ein doppelwerthiges Element darstelle. Es liegt nun nahe, auch für Ascaris anzunelmen, dass die Chromosomen der Somakerne „Theilungselemente niedrigerer Ordnung" darstellen, deren Anzahl in einem gesetzmässigen Verhältniss zur Anzahl der in den Stammzellkernen auftretenden Schleifen steht. Während bei Cyclops dieses Verhältniss durch 1:2 ausgedrückt werden kann, ist der Nenner dieses Bruches bei Ascaris vielleicht eine etwas grössere Zahl. Während also die Theilung der Urgenitalzelle von Cyclops streng genommen eine "Kerntheilung mit bivalenten Elementen" oder kürzer eine „bivalente Kerntheilung“ ist, hätten wir in den Theilungen der Stammzellen von Ascaris vielleicht eine wirkliche „plurivalente Kerntheilung“" vor uns.

In ähnlichem Sime bat sich vo n Wa si elew sky ${ }^{1}$ ) bezüglich der Theilungen ausgesprochen, welche in der Keimzone der Genitalschläuche von Ascaris meg. auftreten. Er beobachtete auch hier einen Zerfall des Chromatinfadens in eine Anzahl (8-10) von Theilungseinheiten niedrigerer Ordnung, welche die Gestalt yon cubischen Elementen haben. „Dieser Theilungsmodus beweist, dass der Chromatinfaden durch Verschmelzung einer grösseren Anzahl von Chromatinkörpern entstanden ist." Ich werde

1) v. Wasielewsky, Die Keimzone in den Genitalschläuchen von Ascaris megalocephala. Arch. f. mikr. An, 41. Bd. 1893. (13.) 
weiter unten auf die ron Wasielewsky'schen Untersuchungen nochmals zurickkommen.

Es wurde im Obigen gezeigt, dass sow ohl bei Cyclops wie bei Ascaris die Urgeschlechtszellen sich durch die geringere Zahl und die bedeutendereGrösseder chromatischen Elemente ron den somatischen Kernen unterscheiden. Die Art und Weise, wie die Trennung des somatischen und generativen Kerntheilungsmodus während der Furchung erfolgt, ist jedoch bei den beiden Formen eine verschiedene.

Bei Cyclops stellt sich die erste Furchungstheilung als plurivalente (heterotypische) Mitose dar (3, Fig. $3 \mathrm{c}$ ). Die zu Ringen geschlossenen Doppelfadensegmente ordnen sich ,p a rallel zur Spindelaxe und es kommt auf diese Weise eine typische Tonn en form zu Stande." Die Anzahl der je aus vier Elementen zusammengesetzten Ringe ist halb so gross, als die "normale" Theilungszahl (8) der chromatischen Elemente (3, S. 21 [180]). An jeden Pol rücken vier Halbringe, deren jeder ein doppelwerthiges Element darstellt. Während der folgenden Furchungsstadien treten, wie ich mich bei verschiedenen Cyclops-Species üherzeugte, in sämmtlichen Kernen wieder einfache Theilungselemente auf: bei jeder Theilung rticken an jeden Pol je a cht einf a che schleifenförmige Chromosomen. Doch unterscheiden sich namentlich die während der ersten Furchungsstadien zu beobachtenden Theilungen in einigen Punkten von den "somatischen" Mitosen, wie sie später bei der Differenzirung der Keimblätter gefunden werden. Die Schleifen sind grösser und unregelmässiger gelagert, als bei den letztgenannten Mitosen. Bei Polansicht stellt der Aster (Mutterstern) keine regelmässige kranzförmige Figur dar, vielmehr sind die Schleifen gleichmässig über die ganze Aequatorebene vertheilt (Taf. XXXII, Fig. 1a). Bei Seitenansicht sieht man häufig einzelne Schleifenenden weit hervorragen (Fig. 1 b) und dies erinnert an die Bilder, welche nach Fle m m in $\mathrm{g}^{1}$ ) die heterotypische Theilung im Salamanderhoden und ebenso die Theilung der Urgenitalzelle von Cyclops dar-

1) W. Flemming, Neue Beiträge zur Kenntniss der Zelle. Arch. f. mikr. An. 29. Bd. 1887. (14.) 
bietet. Es kommt noch hinzu, dass das Asterstadium ${ }^{1}$ ) und die Metakinese relativ häufig, der Dyaster dagegen seltener auftritt, während umgekehrt bei den eigentlichen somatischen $\mathrm{Mi}$ tosen die Dyasterfiguren verhältnissmässig zahlreich sind. Bei den Theilungen der Furchungsstadien muss demnach die Metakinese (F l e m m in $\mathrm{g}$ ) relativ lange dauern und auch hierin zeigt sich eine Annäherung an die heterotypische Mitose (vgl. 3, S. 4, sowie F le m ming, 14, S. 408). Ich möchte die Kerntheilungen der ersten Furchungsstadien als embryouale Mitosen den generativen (plurivalenten, heterotypischen) ${ }^{2}$ ) und somatischen Mitosen gegenüberstellen. In Bezug auf die Anzahl der Theilungselemente stimmen die embryonalen Mitosen mit den somatischen überein, in ihrem Habitus zeigen sie dagegen vielfach Anklänge an die generativen Theilungen.

Im Verlauf der weiteren Furchungsvorgänge nehmen die embryonalen Mitosen mehr und mehr den somatischen Typus an: Die Elemente werden kürzer, ihre Anordnung gewinnt an Regelmässigkeit, die Daner der Metakinese wird eine geringere und das Dyasterstadium nimmt dafür eine längere Zeit in Anspruch.

Zur Zeit der Differenzirung der Keimblätter sieht man dann auf einmal wieder neben dem somatischen Typus Kerntheilungsbilder auftreten, welche den bei der ersten Furchungstheilung beobachteten ibberaus nahe stehen und sich dem plurivalenten Schema einfügen (Fig. 6). Diese Bilder finden sich bei der Theilung der Urgenitalzelle (A), nachdem schon bei der vorhergehenden Theilung, aus welcher die A- und B-Zelle hervorgegangen waren, wieder längere Schleifen bemerkbar waren.

1) Ich unterlasse es, an Stelle der Bezeichnungen "Aster" und "Dyaster" die Ausdrücke "Astroid" und "Dyastroid" anzuwenden, welche Flemming neuerdings in Beantwortung eines Einspruchs von Fol in Vorschlag gebracht hat (Zur Nomenklatur der Zelltheilung, Anat. Anz., 7. Jahrg. 1892, 15). Tch glaube, dass speziell die ältere Flemming'sche Bezeichnung Dyaster so geläufig und bequem ist; dass sie ohne zwingenden Grund nicht aufgegeben werden sollte.

2) Die Ausdrücke generativ und plurivalent decken sich nicht vollkommen, da vom $\mathrm{R}$ at h auch in der Urniere der Embryonen und Larven von Salamandra und ebenso in den Dotterkernen im Bereich des Mitteldarms von jungen Larven Mitosen gefunden hat, die wohl als plurivalent $z u$ deuten sind $(\mathbf{1}$, S. 106). Nach H. E. Ziegler (6a) wäre auch die halbe Zahl der Chromosomen in den Merocytenkernen der Selachier in diesem Sinn zu erklären. 
Im Gegensatz zu Cyclops wird bei Ascaris der generative Typus in unmittelbarer Descendenz von der befruchteten Eizelle auf die Stammzellen und Urgeschlechtszellen übertragen, während die Ursomazellen direkt zum somatischen Typus überspringen. Dabei vollzieht sich nach Boveri in denselben ein eigenthümlicher Differenzirungsprocess, auf welchen ich im nächsten $\mathrm{Ab}$ schnitt zurïckkommen werde. Hier soll nur noch einmal hervorgehoben werden, dass sich also bei Cyclops und Ascaris die Differenzirung des generativen und somatischen Typus insofern verschieden gestaltet, als dort der erstere während der Furchungsstadien gewissermaassen latent bleibt, während er sich bei Ascaris in kontinuirlicher Folge von der befruchteten Eizelle bis zar Urgeschlechtszelle forterhält.

Während so Ascaris ein viel prägnanteres Beispiel für den kerngeschichtlichen Gegensatz zwischen Geschlechts- und Somazellen darstellt, dürften die Befunde bei Cyclops vielleicht in anderer Richtung von Interesse sein. Es seheint mir, dass durch dieselben der Zusammenhang zwischen den beiden Kerntheilungsformen, wie sie sich bei Ascaris in extremer Weise einander gegenüberstehen, vermittelt und das physiologische Verständniss dieser Verschiedenheiten angebahnt wird. Es wurde bereits oben darauf hingewiesen, dass der e mbry on al e Kerntheilungstypus im Verlauf des Furchungsprocesses allmählich in den somatischen Typus übergeht. Andrerseits zeigt derselbe nicht nur in der Grösse der Elemente, sondern vor Allem auch in der Bewegungsweise derselben Anklänge an die generative (plurivalente) Form. Die Anordnung der Chromosomen, wie sie auf Schnittbildern zum Ausdruck kommt, lässt nämlich einen Rückschluss zu auf die Bewegungsvorgänge, welche zur schliesslichen Orientirung der Theilungselemente führen: es kann jedenfalls soviel gesagt werden, dass die Bewegungsvorgänge lebhaftere und unter dem Spiel einander entgegenwirkender Richt- und Torsionskräfte komplicirtere sein müssen, als dies bei den somatischen Mitosen der Fall ist. Genau dieselbe Erscheinung tritt aber in viel auffalligerem Maasse in den generativen (plurivalenten) Mitosen, speciell bei der eigentlichen heterotypischen Form hervor, wie dies schon von Flemming ausgeführt und auch von mir wiederholt hervorgehoben worden ist. In Bezug auf die Grösse der Elemente und ebenso auf die Bewegungsweise derselben kann also der 
embryonale Typus auch mit dem generativen durch vielfache Uebergänge in Zusammenhang gebracht werden.

Immerhin wäre aber noch ein Unterschied zwischen dem embryonalen und generativen Modus hervorzuheben. Bei der generativen (plurivalenten) Mitose treten doppelwerthige Elemente in der halben Anzahl auf, bei den embryonalen Mitosen dagegen ist bereits die Normalzahl einwerthiger Elemente vorhanden. Aber auch in diesem Punkte dürfte kein untiberwindlicher Gegensatz zwischen den beiden Typen bestehen: wir kennen einerseits Fälle, wo beim plurivalenten Typus im Dyaster eine nachträgliche Zerfällang der Doppelelemente in einwerthige erfolgt, z. B. die Theilung der Urgenitalzelle von Cyclops, andererseits gibt es, wie bereits oben (S. 764, Anm. 2) hervorgehoben wurde, embryonale (nicht-generative) Mitosen, die nur die halbe Anzahl von Theilungselementen zeigen und vermuthlich als plurivalente Theilungen aufzufassen sind.

Alles in allem können wir demnach eine zusammenhängende Reibe aufstellen, welche von den somatischen Mitosen durch die embryonalen hindurch zu den generativen führt. Diese Reihe ist gekennzeichnet durch die allmähliche Grössenzunahme der Elemente und die lebhafteren und komplicirteren Bewegungsvorgänge, welche sich bei der Rangirung derselben abspielen. Im Zusammenhang damit dürfte ein andres physiologisches Moment stehen: je mehr wir uns dem echten generativen Typus nähern, um so mehr nimmt die Tendenz zur letzten Zerfällung der Doppelchromosomen in einwerthige Elemente $a b$, so dass sie schliesslich bei den echten plurivalenten Mitosen überhaupt unterbleibt, wenn auch offenbar immer noch eine gewisse Neigung daza vorbanden ist (3, S. 10 [169], 4, s. 483).

Wir kommen so zu dem auffallenden Schluss, dass, je grösser die Elemente sind, um so weniger leicht die endgültige Zerfällung. der Doppelelemente zu Stande kommt. Damit steht vielleicht die von Boveri (12, S. 125) erwähnte Erscheinung im Zusammenhang, dass bei Ascaris häufig noch im Vierzellenstadium die ursprünglichen zwei Schleifen in allen vier Furchungskugeln erhalten bleiben. Die Grösse der Kerne und die Masse der Elemente scheint hier - unter gewissen, nicht kontrollirbaren Umständen der Zerlegung der Schleifen in Elemente niedrigerer Ordnung im Wege zu stehen. 
Andrerseits zeigen nach von Wasiclewsky die kleinen Kerne der Keimzone (trotzdem sie im Cyclus der generativen Zellen liegen) eine Zerlegung des Chromatinfadens in Elemente niedrigerer Ordnung. Es würde dies nach dem Obigen mit der relativ geringeren Masse der chromatischen Substanz in Verbindung zu bringen sein.

Wenn so die generativen Mitosen mit den embryonalen und damit auch mit den somatischen durch Uebergänge verbunden sind, so ist doch nicht ausser Acht zu lassen, dass wenigstens zwischen den beiden Extremen ein bemerkenswerther Unterschied besteht. Ich möchte es wenigstens nicht für unbedeutsam halten, dass bei den plurivalenten Theilungen gerade die letzte Segmentirung unterbleibt ${ }^{1}$ ). Boveri hat aus seinen Befunden den Schluss gezogen, dass das bandförmige Chromosom von Ascaris megalocephala nicht etwa eine continuirliche Serie gleichwerthiger Bestandtheile darstellt, sondern dass es eine symmetrische Bildung ist, deren Endabschnitte sich ganz anders verhalten als die centralen (12, S. 122). Wenn ich auch die Erscheinungen, auf welche Boveri seinen Schluss gründet, nicht als normal betrachten kann, so glaube ich doch angesichts des Auftretens von doppelwerthigen Elementen und der augenscheinlichen Besonderheiten der letzten Segmentirung, dass die Frage nach dem symmetrischen Bau der (Doppel-)Chromosomen eine weitere Beachtung verdient. Es würde hier vielleicht noch in Betracht zu ziehen sein, dass nach allem, was wir davon wissen, in der zweiten Reifungstheilung das Chromatin der Doppelelemente in einwerthige Elemente zerlegt und so auf die beiden Tochterkerne vertheilt wird, ein Vorgang, der gleichfalls auf einen symmetrischen Bau der Doppelelemente hinweist.

Es soll nun noch der andre Punkt besprochen werden, in welchem nach B overi die kerngeschichtliche Differenzirung der Geschlechts- und Somakerne zum Ausdruck kommt, nämlich die eigenthümliche Abspaltung der Schleifenenden. Ich werde ver-

1) Ascaris bildet hier vielleicht eine Ausnahme, insotern hier die Schleifen der Keimbahn-Mitosen mehr als zwei Elementen der SomaMitosen entsprechen (s. S. 772 unten). Vergl. die Befunde von $B o v e r i$ und von Wasielewsky. 
suchen, den Nachweis zu führen, dass es sich hier nur um eine pathologis $\mathrm{che}$ Erscheinung handeln kann.

Es sind mir selbst bis jetzt von Ascaris keine späteren Stadien als das Vierzellenstadium zu Gesicht gekommen und ich vermag daher die Boveri'schen Angaben bei Ascaris selbst nicht zu kontrolliren. Nur so viel möchte ich gleich hier bemerken, dass schon in den Anfangsstadien der Furchung vielfach augenscheinlich pathologische Bilder vorkommen. Boveri hat diesen Verhältnissen auch Rechnung getragen und bemerkt daher (5, S. 697), dass er die vollkommen lebendigen Würmer persönlich dem Darm des vor seinen Augen geschlachteten Pferdes entnommen hat, und er glaubt mittelst Abtödtens der Eier durch Hitze zuverlässige Präparate erhalten zu haben. Nun ist aber dem gegenüber in Betracht zu ziehen, dass zwischen der Tötung des Pferdes und dem Abdecken und Oeffnen desselben immerhin eine beträchtliche Zeit, mindestens zwanzig Minuten, zu vergehen pflegt, ein Zeitraum, welcher genügen würde, um den normalen Gang der Furchungstheilungen zu stören. Es ist aber des weiteren auch nicht ausgeschlossen, dass die Würmer bereits im lebenden Pferd unter nicht ganz normalen Bedingungen gelebt haben. Beispielsweise liesse sich denken, dass eine beim Wirth aufgetretene Verdauungsstörung für den Parasiten Ernährungsanomalien herbeiführt. Dies würde sich aber leicht in degenerativen Veränderungen der Geschlechtsprodukte äussern können ${ }^{1}$ ).

In den auf zahlreichen Präparaten mir vorliegenden Zweiund Vierzellenstadien von Ascaris megalocephala bivalens habe ich wohl vielfache Unregelmässigkeiten, dagegen keine Furchungsbilder finden können, welche auf die von Boveri angegebenen Differenzirungsvorgänge hingewiesen hätten. Aeltere Stadien fehlen mir.

Dagegen besitze ich eine Anzahl von Präparaten von einem andern Objekte, welche ganz analoge Kerntheilungsfiguren zeigen

1) Es kommt hier die den hiesigen Pferdeschlächtern geläufig'e Thatsache in Betracht, dass Ascaris vorzugsweise bei jungen, gesunden und gut genährten Thieren gedeiht und in Menge vorkommt. Die zum Schlachten verwendeten Pferde sind aber sehr häufig ältere und abgetriebene, wenn nicht sogar kränkliche Thiere, und es ist dann stets mit der Möglichkeit zu rechnen, dass die in denselben etwa vorkommenden Ascaris auf dem Aussterbeetat stehen oder wenigstens nicht unter vollkommen zusagenden Bedingungen leben. 
und für welche der Nachweis, dass es sich hier um pathologische Veränderungen handelt, mit Bestimmtheit erbracht werden kann. Im Mai 1890 konservirte ich eine in einem Tümpel am Rhein in grosser Menge vorkommende Cyclops-Spezies (Cyclops strenuus), und zwar theils unmittelbar an Ort und Stelle, theils, nachdem die Thiere ein Zeit lang in Aquarium gelebt hatten. Ebenso besitze ich konservirtes Material vom Mai 1891. Dagegen fand ich die Spezies in den folgenden Jahren in der betreffenden Jahreszeit nicht wieder. Es liegen mir fast alle Furchungsstadien mehrfach vor und es liess sich feststellen, dass jedenfalls alle 2-, 4-, 8- und 32-Zellenstadien in allen Furchungszellen Kerntheilungen vom gewöhnlichen "embryonalen" Habitus (Fig. 1) aufweisen. Von einer Differenzirung der Geschlechtszellen konnte ich in diesen früheren Furchungsstadien nichts bemerken, trotzdem ich eine grosse Anzahl von Eiern, Kern für Kern, sorgfältig durchmusterte. Unter drei Präparaten, welche das Sechzehnzellenstadium zeigen, wiesen nun aber zwei Kerntheilungsbilder auf, welche eine auffallende Aehnlichkeit mit den B overi'schen Bildern besitzen. Ich vermuthete schon bei der ersten Untersuchung, dass ich es mit pathologischen Bildern zu thun habe, um so mehr als ich in dem abnorm sich furchenden $\mathrm{Ei}$ einer Meduse, Aequorea, auf entsprechende Kerntheilungsformen stiess. Inzwischen habe ich, durch die Boveri'schen Publikationen veranlasst, eine genauere Untersuchung vorgenommen und bin dabei zu dem Ergebniss gelangt, dass es sich hier thatsächlich um Degenerationsformen handelt ${ }^{1}$ ).

Die Figur 2 zeigt einen Schnitt durch ein Sechszehnzellenstadium. S ä $\mathrm{mmtl} \mathrm{i} \mathrm{ch}$ e Kerntheilungsfiguren sind dadurch ausgezeichnet, dass die Theilungselemente in der normalen $\mathrm{An}$ $\mathrm{z}$ a hl auftreten, dass aber der Aequator von einem Kranz uberzähliger Chromatimpartikelchen umstellt ist. Dies lässt sich im Einzelnen an der Figurenreihe Fig. 3, a-f verfolgen, welche die wichtigsten Stadien vom Asterstadium bis zum Dispirem zeigt. Die Abspaltung der Chromatinpartikelchen erfolgt offenbar zur

1) Asterstadien mit abgesprengten oder verirrten Chromosomen hat D. Hansemann auch in Carcinomen und Sarcomen gefunden. Die betreffenden Figuren, Taf. X, Fig. 17-22, entsprechen ziemlich genau der Figurenreihe, Fig. 3a-f. Vergl. D. Hansemann, Ueber pathologische Mitosen. Virchow's Archiv. Bd. 123. 1891. (16.) 
Zeit, wenn sich die Schleifen zum Aster ordnen. Sie liegen anfangs in der ganzen Kernperipherie, später im Aequator derSpindel, wie dies namentlich in den charakteristischen Dyasterstadien besonders auffällig ist (Fig. 3, d). Im Dispiremstadium (Fig. 3, f) liegen dann die Partikelchen einseitig in einiger Entfernung vom Kern und zwar zwischen demselben und der Eiperipherie, welch' letztere jedem einzelnen Kern gegenüber eine muldenförmige Vertiefung zeigt. Ich möchte gleich hier auf die Achnlichkeit hinwiesen, welche speciell zwischen dem Dyasterstadium Fig. 3, d und der Boverischen (halbschematischen) Figur 8 (linke Furchungszelle) im Artikel „Befruchtung" (11) besteht.

Das dritte meiner Präparate, welches das Sechzehnzellenstadium aufweist, zeigt die meisten Kerne in Ruhe (Fig. 4, a). Bei der angewandten Methode (erwärmte Fle mming'sche Flüssigkeit, Stückfürbung in Boraxkarmin, Nachfärbung in Hämatoxylin) heben sich die rothgefärrbten Nucleolen in sehr schöner Weise von dem blaugefärbten Chromatingerüst ab. Irgend etwas Besonderes war an diesen ruhenden Kernen nicht zu beobachten. Danebẹn traten einzelne Kerne im Asterstadium auf, in welchen insofern eine Andeutung des oben beschriebenen Vorganges zu bemerken war, als einzelne wenige Chromatinpartikelchen herausgeschleudert waren (Fig. 4, b). An der Färbung liess sich leicht feststellen, dass es sich wirklich um chromatische, und nicht um nucleoläre Substanz handelt. Ausser den erwähnten Kernbildern waren aber noch eine Anzahl vollkommen normaler Asterstadien und - was besonders ausschlaggebend ist - einige durchaus normale Dyaster (Fig. 4, c) zu beobachten. Von einer Umstellung des Aequators mit Chromatinpartikelchen war an denselben nichts zu bemerken.

Wenn schon auf Grund dieses dritten Präparats ein Zweifel hinsichtlich der pathologischen Beschaffenheit der Bilder der beiden andern Präparate kaum möglich ist, so kann ich zur weiteren Bekräftigung meiner Auffassung noch anführen, dass mir von meinen entwicklungsgeschichtlichen Untersuchungen her von einer grösseren Anzahl von Copepodenspezies die betreffenden Stadien vorliegen, und dass kein einziges Bild andere, als normale Kerntheilungsformen aufweist. Das Schnittbild Fig. 5 zeigt den Uebergang vom 16- zum 32-Zellenstadium im $\mathrm{Ei}$ von Cyclops brevicornis. Sämmtliche Figuren, im Besondern die ausschlag- 
gebenden Dyaster, zeigen die von andern Mitosen her bekannte Beschaffenheit. Erst in einem späteren Stadium tritt die fruher beschriebene Kerndifferenzirung im Sinne des plurivalenten Schemas auf.

Es kann noch die Frage erhoben werden, warum gerade nur bei Cyclops strenuus die beschriebenen Bilder gefunden worden sind. Es ist vielleicht darauf hinzuweisen, dass diese Art, in unserer Gegend wenigstens, gewöhnlich nur als pelagische Form in grösseren Seen auftritt und daher vielleicht in kleinen Tümpeln and vollends im Aquarium auf weniger zusagende Lebensbedingungen stösst. Die Eier sind daher vielleicht mehr der Invasion der Bakterien und der Einwirkung physikalischer und chemischer Veränderungen der Umgebung ausgesetzt, als dies bei den eigentlichen tümpelbewohnenden Formen der Fall ist.

Wie ans dem Obigen hervorgeht, konnte für eine CyclopsSpezies der Nachweis geführt werden, dass die bei den Furchungstheilungen da und dort anftretende Chromatinabspaltung eine pathologische Erscheinung ist. Nun zeigen aber die bei Cyclops auftretenden abnormen Theilungsfiguren der Hauptsache nach eine grosse Aehnlichkeit mit denjenigen, welche von $\mathrm{Bo}$ veri für Ascaris als normale Vorkommnisse beschrieben worden sind.

Es ist eine der Aufgaben des vorliegenden Aufsatzes gewesen, den Nachweis zu erbringen, dass hinsichtlich der bei der Eibildung, bei der Eifurchung und Keimblätterbildung auftretenden Kerntheilungsformen innerhalb des ganzen Metazoenreiches eine ausserordentliche Gleichförmigkeit und Gesetzmässigkeit besteht. Es war möglich, dje bei der Bildung der Geschlechtsproducte und bei der Differenzirung der Geschlechtszellen auftretenden scheinbar heterogenen Theilungstypen unter Zugrundlegung des plurivalenten Schemas auf einander und auf die gewöhnlichen Formen der Mitose zurückzuführen. Man steht also vor der Wahl, entweder den bei Ascaris beobachteten Bildern eine Ausnahmestellung zuzuweisen und das, was für Cyclops als pathologisch nachgewiesen werden konnte, bei Ascaris als normal gelten zu lassen, oder aber man muss annehmen, dass dieser Theil der Boveri'schen Untersuchungen auf einem Irrthum beruht. Ich bin weit davon entfernt, die Möglichkeit abzuweisen, dass zwischen zwei sich entfernt stehenden Formen eine der- 
artige Verschiedenheit bestehen könnte, aber ich möchte angesichts der schwerwiegenden Einwände, die sich gegen B overi's Bilder zu Felde führen lassen, es als äusserst wahrscheinlich betrachten, das die Chromatinabspaltung bei der Furchung von Ascaris eine pathologische Erscheinung ist.

Ich möchte demnach die Annahme vertreten, dass bei Ascaris thatsächlich die Somazellen andere Kerntheilungsbilder darbieten als die Zellen der "Keimbahn", insofern in den ersteren bei jeder Theilung eine Zerfällung der Schleifen in Elemente niedrigerer Ordnung erfolgt, dass aber die Chromatinabspaltung ein Accidens pathologischer Na$t u r$ ist.

Es könnte nun der Einwand erhoben werden, dass es doch auffallend wäre, dass gerade die Zellen der Keimbahn normal bleiben, während die Kerne anderer Zellen pathologischen Veränderungen anheimfallen. Es wurde aber oben gezeigt, dass bei der plurivalenten Kerntheilung die Wirksamkeit der ordnenden und richtenden Kräfte eine andre sein mnss, als dies bei den gewöhnlichen Mitosen dẹr Fall ist, dass also in dieser Richtung eine physiologische Verschiedenheit zwischen beiden Kerntheilungsformen bestehen muss. Es wäre denkbar, dass in dieser physiologischen Verschiedenheit auch eine verschiedene Widerstandskraft der plurivalenten Mitosen der Keimbahn gegen äussere Einflüsse bedingt ist.

Nehmen wir es als richtig an, dass die Chromatinabspaltung ein pathologischer Vorgang ist, so wird doch damit den Bov e r i 'schen Befunden ihre Bedeutung nicht genommen: dieselben stellen vielmehr ein ausserordentlich werthvolles Seitenstück zu den Beobachtungen bei Cyclops dar, insofern bei ihnen viel früher ein kerngeschichtlicher Untersehied zwischen Geschlechts- und Somazellen auftritt.

Es ist in dem vorliegenden Aufsatz der Versuch gemacht worden, für einige früher ausgesprochenen Sätze die Giltigkeitsgrenzen nach der einen und andern Richtung hin weiter zu stecken. Dies durfte geschehen, da sich inzwischen das einschlägige empirische Material bedeutend vermehrt hat und da andrerseits, so viel ich sehe, keine Thatsache bekannt geworden 
ist, welche in unlösbarem Widerspruch mit der hier vertretenen Anschauungsweise steht. Man könnte nun die Frage erheben, was denn eigentlich mit der Aufstellung des plurivalenten Schema's erreicht ist?

Es muss hier zunächst zugegeben werden, dass ein weiterer Einblick in die Mechanik der Kerntheilung nur bis zu einem gewissen Grade gewonnen ist. Denn in dem plurivalenten Sehema soll einzig und allein die Behauptung zum Ausdruck kommen: der Chromatinfaden aller Kerne hat die Tendenz, vor jeder Theilung in eine für die Spezies charakteristische Anzahl von Segmenten zu zerfallen. Dieser Zerfall kommt aber unter besonderen Verhältnissen nur in unvollständiger Weise zur Durchführung, so dass die Segmentirung nur bis zur Bildung von Theilungseinheiten höherer Ordnung, speciell von Doppelelementen fortschreitet ${ }^{1}$ ).

Ein Vergleich der "plurivalenten" Mitosen mit denjenigen des "embryonalen" Typus liess es ferner als wahrscheinlich erscheinen, dass die Fähigkeit der chromatischen Substanz, sich in Einheiten niedrigerer Ordnungu zerlegen, in einem gewissen Abhängigkeitsverhältniss zur Massedes Chromatins und damit zur Grösse des Kerns steht. Je grösser der Kern und damit die Masse der chromatischen Substanz ist, um so weniger leicht kommt die endgtiltige Zerfällung der Doppelelemente zu Stande. Darauf weisen die Befunde "plurivalenter" Mitosen in embryonalen und larvalen Geweben von Salamandra ( $v$ om Rath), sowie das ausnahmsweise Vorkommen solcher in beiden Zellen des Zweizellen-

1) Die Tendenz des Chromatinfadens, sich auch in diesem Falle in die "Normalzahl“ von Elementen zu zerlegen, dürfte in zahlreichen Besonderheiten der plurivalenten Theilungsformen zum Ausdruck kommen. Dahin gehört z. B. in der Urgenitalzelle von Cyclops die Herzform der Elemente während der Prophasen und der sekundäre Durchbruch derselben im Dyaster (3, S. 10 [169]). Vielleicht sind hier noch anzuführen die schon von Flemming beschriebenen Krümmungen und Verschlingungen der Schleifen bei der heterotypischen Theilung, sowie die Ueberkreuzungen und Achterbildungen, welche die Doppelfadeuzüge vor der ersten Richtungstheilung zeigen. „Es ist, als ob darin sich ein unbefriedigtes Bestreben der doppelwerthigen Elemente äussern würde, an den präformirten Stellen doch noch schliesslich zum Durchbruch zu gelangen und sich so in einwerthige Elemente zu zer$\operatorname{legen}^{*}(4$, S. 483). 
stadiums von Ascaris (Boveri) und andrerseits die Befunde in der Keimzone von Ascaris (von Wasielewsky) hin.

Der Umstand endlich, dass (wenigstens in den meisten Fällen) gerade nur die letzte Segmentirung unterbleibt, lässt es als möglich erscheinen, dass innerhalb des Chromatinfadens die beiden zu einem Doppelelement gehörigen Elemente (Idanten) in engerer Beziehung zu einander, als zu den benachbarten Elementen des Fadens stehen und dass demnach der letzten Segmentirung eine besondere Bedeutung zukommt im Vergleich zu denjenigen Segmentirungsprocessen, welche eine Zerlegung des Chromatinfadens in Doppelelemente herbeigeftihrt haben.

Auf eine besondere Bedeutung der letzten Segmentirung: scheint auch die Rolle hinzuweisen, welche dieser Vorgang bei der zweiten Reifungstheilung spielt. Nach Allem, was wir wissen, ist es nämlich höchst wahrscheinlich, dass bei der zweiten Reifungstheilung die letzte Segmentirung (Quertheilung), also die Zerfällung der Doppelelemente in einwerthige Elemente (Metalyse) diejenige Rolle spielt, welche bei allen andern Mitosen der Längsspaltung zukommt. Während bei letzteren die zwei durch Längsspaltung eines Mutterelements entstandenen Schwesterelemente sich je auf einen Tochterkern vertheilen, scheint dies bei der zweiten Reifungstheilung für die beiden durch $\mathrm{Quer-}$ theilung eines Doppelelements entstandenen einwerthigen Elemente zu gelten.

Die vorstehenden, auf die Theilungsmechanik bezulglichen Ergebnisse, tragen zum grossen Theil einen hypothetisehen Charakter. Aber ich glaube doch, den Vorstellungen, zu welchen ich bei meinen mehrjährigen Untersuchungen auf dem Gebiet der generativen Kerntheilungen allmählich gelangt bin, hier Ausdruck geben $\mathrm{zu}$ sollen, weil ich meine, dass denselben eine gewisse Einheitlichkeit und Zusammengehörigkeit nicht abgesprochen werden kann.

Jedenfalls glaube ich, meiner früher $(\mathbf{3}, \mathbf{4})$ ausgesprochenen Behauptung, dass im Cyclus der generativen Zellen Kerntheilungen mit doppelwerthigen Elementen (plurivalente Mitosen) eine weit verbreitete Erscheinung sind, in folgenden drei Sätzen eine erweiterte und besser begrindete Form geben zu können:

1) Die chromatische Substanz des Kernes besitzt die Tendenz, vor jeder Theilung durch Segmentirung (Quertheilung) in 
T a b elle.

\begin{tabular}{|c|c|c|c|}
\hline & Nicht-generative Mitosen. & Generative Mitosen. & \\
\hline  & $\begin{array}{l}\text { Somatische Mitosen mit der } \\
\text { Normalzahl von Elementen. } \\
\text { Embryonale Mitosen mit } \\
\text { der Normalzahl von Elemen- } \\
\text { ten. Die Schleifen zeigen in } \\
\text { Grösse und Anordnung An- } \\
\text { klänge an die generativen } \\
\text { Mitosen. }\end{array}$ & $\begin{array}{l}\text { Mitosen der Keimzone von } \\
\text { Ascaris. Zerfall der Schleifen } \\
\text { in zahlreiche Elemente nie- } \\
\text { drigerer Ordnung (von Wa- } \\
\text { sielew s ky). }\end{array}$ & \\
\hline 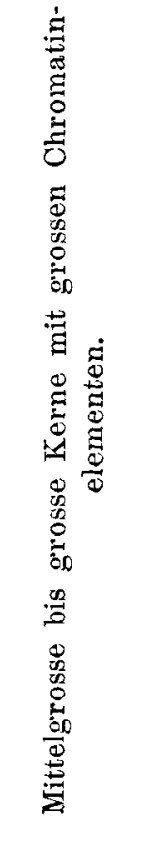 & $\begin{array}{l}\text { Embryonale Mitosen mit } \\
\text { der Hälfte der Normalzahl. } \\
\text { ? Doppelwerthigkeit der Ele- } \\
\text { mente (embryonale und lar- } \\
\text { vale Gewebe von Salaman- } \\
\text { dra, vom Rath). }\end{array}$ & $\begin{array}{l}\text { Erste Theilung der Rei- } \\
\text { fungsphase. Der Chromatin- } \\
\text { faden zerlegt sich in der } \\
\text { Normalzahl von Elementen. } \\
\text { Je zwei derselben bleiben } \\
\text { aber in engerem Zusammen- } \\
\text { hang (v o m Rath, Häck er). } \\
\text { Urgenitalzelle vonCyclops. } \\
\text { Je zwei Elemente bleiben mit } \\
\text { einander verbunden. Im Dy- } \\
\text { aster erfolgt nachträglich die } \\
\text { Zerlegung in einwerthige } \\
\text { Elemente (H ä cker). } \\
\text { Heterotype und homöotype } \\
\text { Theilungen im Salamander- } \\
\text { hoden. ? Je zwei Elemente } \\
\text { sind mit einander verbunden } \\
\text { und bleiben dies auch im Dy- } \\
\text { aster (Hä ck er, vom Rat } \mathrm{h} \text { ). } \\
\text { Generative Theilungen bei } \\
\text { Ascaris. ? Mehr als zwei Ele- } \\
\text { mente niedrigerer Ordnung } \\
\text { bleiben mit einander ver- } \\
\text { bunden. }\end{array}$ & 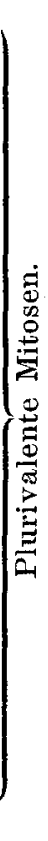 \\
\hline
\end{tabular}

eine für die Spezies charakteristische und konstante Anzahl von Theilungselementen (Idanten) zu zerfallen (normale Theilungszahl) ${ }^{1}$ ).

2) In grossen, chromatinreichen Kernen, wie solche während der Furchung und späteren Embryonal- und Larvalentwicklung auftreten, unterbleibt zuweilen die letzte Segmentirung des Fa-

1) Ich habe früher versucht, nachzuweisen, dass es sich in der Regel un einfache, auf den Primzahlen 2 und 3 sich aufbauende Zahlenverhältnisse handelt. Vergl. V. Häcker, Die Furchung des Eis von Aequorea Forskalea. Arch. f. mikr. Anat. 40. Bd. 1892. (17.) 
dens, so dass doppelwerthige Elemente auftreten (Befunde bei Embryonen und Larven von Salamandra).

3) Der Ausfall oder wenigstens die unvollkommene Durchführung der letzten Segmentirung ist eine weitverbreitete Erscheinung im Cyklus der generativen Zellen (plurivalente Theilungen). Im Besondern sind in diesem Sime die Vierergruppen der Reifungstheilungen als längsgespaltene Doppelelemente zu betrachten.

Freiburg i. Brsg., den 12. Februar 1894.

\section{Erklärung der Abbildungen auf Tafel XXXII.}

Sämmtliche Figuren sind nach Schnitten gezeichnet, Fig. 1 u. 3 mit Zeiss, hom. Imm. $3 \mathrm{~mm}$, Oc. 8 (Vergr. 667), Fig. 2 und $4-6$ mit Obj. D, Oc. 4 (Vergr. 435).

Fig 1. „Embryonale" Mitosen aus den exsten Furchungsstadien des Eies von Cyclops strenuus. a Polansicht des Asterstadiums. b Seitenansicht desselben. Einzelne Fadenenden ragen weit über das Niveau des Aequators heraus.

Fig. 2. Sechszehnzellenstadium von Cyclops strenuus. Pathologische Chromatinabspaltung. Die Buchstaben weisen auf die Einzelbilder der Fig. 3 hin, in welchen die betreffenden Stadien (theilweise nach andern Schnitten) in vergrössertem Maassstab wiedergegeben sind.

Fig. 3. Pathologische Kerntheilungsformen aus dem sich furchenden Ei von Cyclops strenuus. a Asterstadium in Seitenansicht. b Dasselbe in Polansicht, schräg angeschnitten. c und d Dyasterstadium, die überzähligen Chromatinpartikelchen haben sich in der Peripherie des Aequators angeordnet. e Dyaster in schräger Polansicht. f Zwei Schwesterkerne im Beginn des Dispiremstadiums, die herausgeschleuderten Chromatinpartikelchen liegen der Peripherie des Eies an. An der betreffenden Stelle der Eiperipherie treten muldenförmige Einsenkungen auf.

Fig. 4. Sechszehnzellenstadium von Cyclops strenuus mit Spuren der Chromatinabspaltung. a Ruhende Kerne, völlig normal. b Asterstadium mit einzelnen abgesprengten Chromatinpartikelchen. c Normaler Dyaster. 
Fig. 5. Uebergang vom Sechszehn- in das Zweiunddreissigzellenstadium im Ei von Cyclops brevicornis. Normale Theilungsfig'uren.

Fig. 6. Genitalzellen-Differenzirung bei Cyclops brevicornis. A (ug) Urgenitalzelle, B (um) Urmesodermzelle. Beide sind durch Theilung einer Stammzelle entstanden. Vor (?) der in die Tiefe des Dotters eindringenden Urmesodermzelle liegt eine Gruppe von Entodermzelien, von denen eine in Theilung begriffen ist. In der Urgenitalzelle treten grosse schleifenförmige, in den Entodermzellen kleine winkelförmige Chromatinelemente auf. Das Bild ist zusammenzustellen mit den Boveri'schen Figuren 2 und 4 in 12.

\section{Untersuchungen über das Gehirn der Vögel.}

Von

Dr. F. Brandis (Friedrichsberg, Hamburg).

II. Theil: Das Kleinhirn.

Hierzu Tafel XXXIII.

Das Kleinhirn der Vögel stellt eine bedeutend fortgeschrittene Entwicklungsstufe des Reptilienkleinhirns dar, es bleibt jedoch andererseits weit hinter dem der Säugethiere zurück, hauptsächlich deshalb, weil ihm jene starke Ausdehnung seitwärts fehlt, welche sich dort in der Bildung der Hemisphären äussert. Dem Wurm des Cerebellum der Säuger entspricht das Kleinhirn der Vögel jedoch nur insofern, als es im Gegensatz zu jenem wenigstens theilweise auch die centralen Kerne, welche dort in den Hemisphären gelegen sind, mit enthält. Die Gliederung der Oberfläche des Kleinhirns geschieht in derselben Weise wie bei den Säugern, auch hier gehen von der weissen Substanz des central gelegenen Körpers zahlreiche blattförmige, aus Nervenfasern bestehende Fortsätze ab, die in ihrer Stellung ähnlich den Schaufeln eines Mühlrades angeordnet sind und die Grundlage der Gyri bilden. Sie convergiren sowohl noch dem Centrum, wie auch 
f.



$Q$.



4.

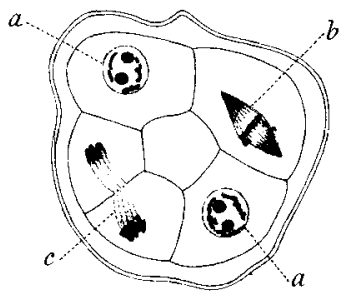

5.

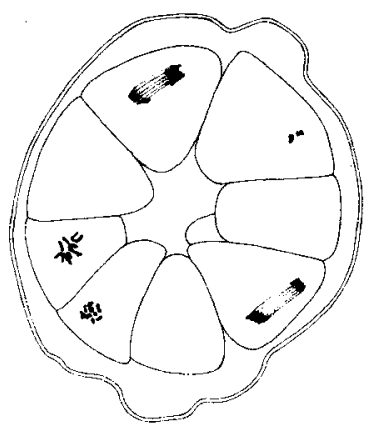

3.
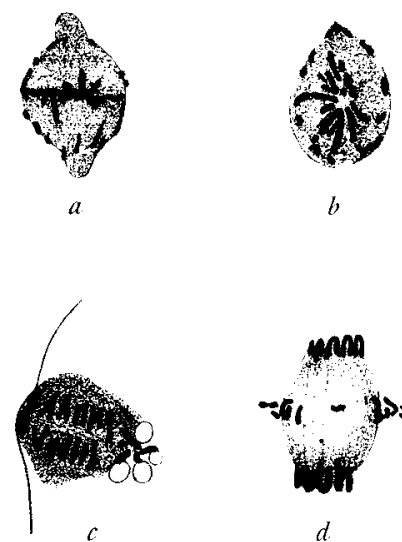

men

$-4 i-1)$

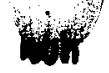

$d$



6.

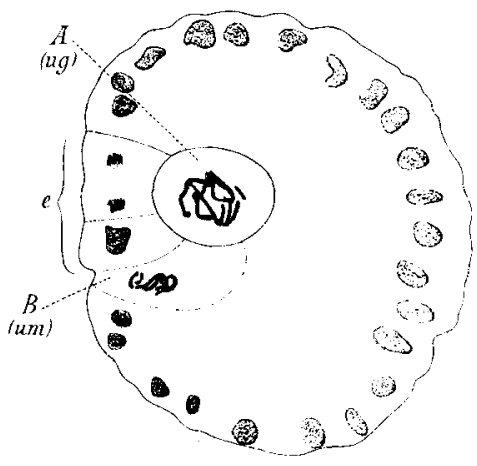

